\title{
Energy-efficient Cluster-based Mechanism for WBAN Communications for Healthcare Applications
}

\author{
Monika Verma \\ M.tech Scholar, Department of Electronics and \\ Eommunication, NIIST, \\ R.G.P.V Bhopal (M.P)
}

\author{
Rajesh Rai \\ Professor, Department of Electronics and \\ Eommunication, NIIST, \\ R.G.P.V Bhopal (M.P)
}

\begin{abstract}
Wireless body area networks (WBANs) are formed with the help of tiny health monitoring sensors on the human body in order to collect and communicate the human personal data. WBANs provides a solution to facilitate the tasks performed in the medical sector, and minimize the chances of errors during the process of medical diagnosis. However, using an expensive key management method is not feasible in highly resource-constrained WBANs. Therefore, we propose and evaluate an energy-efficient key management scheme for WBANs that takes into account available resources of a node during the whole life cycle of key management. Our proposed scheme is a cluster-based hybrid security energy efficient framework. By using multiple clusters, energy-efficiency can be ensured. The performance comparison of our proposed cluster-based key management scheme and low-energy adaptive clustering hierarchy (LEACH)-based key agreement scheme and energy - efficient mechanism shows that the proposed scheme is more energy-efficient, and provides better network lifetime.
\end{abstract}

\section{General Terms}

Medium access layer (MAC), low-rate (LR), PHY, Radio Frequency (RF),DLL

\section{Keywords}

Wireless Body Area Network (WBAN), Energy-efficient, Clustering, IEEE 802.15, LEACH.

\section{INTRODUCTION}

The rapid growth of wireless technologies enables continuous healthcare monitoring of mobile patients using compact biomedical wireless sensor motes. These small easy to wear devices - limited in memory, energy, computation, and communication capabilities - are positioned on a patient; then, they self-configure to form a networked cluster that is capable to continuously monitor vital signs such as blood pressure and flow, core temperature, ECG, oxygen saturation, $\mathrm{CO} 2$ concentration (for respiration monitoring).

Existing technology in the pre- and hospital environment lacks effective methods for compiling information streams, evaluating time dependent trends, managing incomplete data, and providing effective alerts. Hence, we propose a novel wireless communication solution that - with minimum added $\mathrm{RF}$ interference which collects and prioritizes vital signs transmission using WBANs, reliably transfers the acquired patient data to medical terminals or PDAs, and seamlessly supports mobility as patients are moved along different hospital settings.

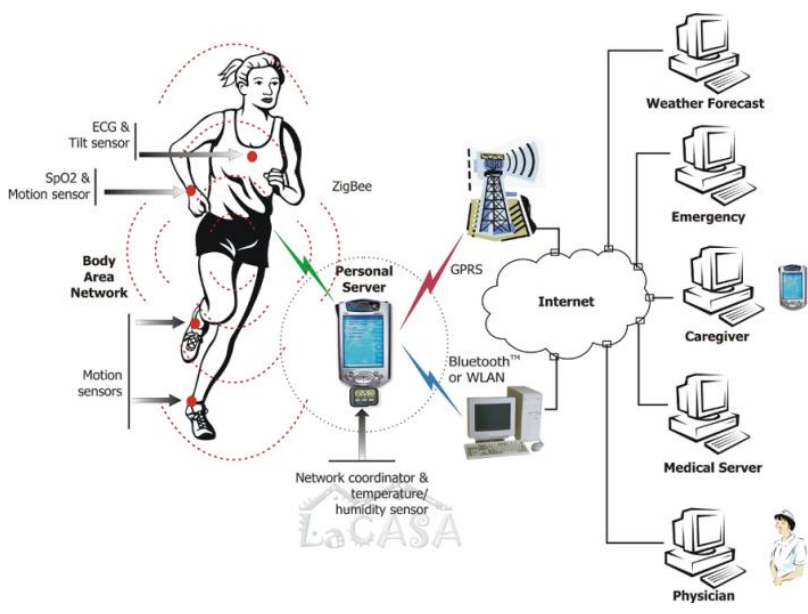

Fig 1 : Proposed Physical Network Architecture

A Body Area Network is formally defined by IEEE 802.15 as, "a communication standard optimized for low power devices and operation on, in or around the human body (but not limited to humans) to serve a variety of applications including medical, consumer electronics / personal entertainment and other". In more common terms, a Body Area Network is a system of devices in close immediacy to a person's body that cooperate for the benefit of the user.

\subsection{Wireless Sensor Network}

A wireless sensor network (WSN) (sometimes called a wireless sensor and actor network (WSAN)) of spatially distributed autonomous sensors to monitor physical or environmental conditions, such as temperature, sound, pressure, etc. and used to cooperatively pass their data through the network to a main location. The more advanced networks are bi-directional, also enabling control of sensor activity.

The WSN is built of "nodes" - from a few to several hundreds or even thousands, where every node is connected to one (or sometimes several) sensors. Each such sensor network node has typically several parts: a radio transceiver with an internal antenna or connection to an external antenna, a microcontroller, an electronic circuit for interfacing with the sensors and an energy source, usually a battery or an embedded form of energy harvesting. LEACH is one of the clustering process used in WSN. LEACH is a stratified protocol in which most nodes transmit to cluster heads, and the cluster heads aggregate and compress the data and forward it to the base station (sink). Each node uses a 
stochastic algorithm at each round to determine whether it will become a cluster head in this round. LEACH concludes that each node has a radio powerful enough to directly approach the base station or the nearest cluster head.

\subsection{Wireless Body Area Network}

Wireless Body Area Network (WBAN) is an exciting technology that assure to bring health care to a new level of personalization. Miniaturized sensors can be put on the body and they can non-intrusively monitor a person's physiological state. Multiple sensors communicate with a mobile phone using wireless interfaces forming a WBAN. WBANs permit monitoring an individual's health continuously in free living conditions, where the individual is free to conduct his/her daily activity.

\section{PATIENTS MONITERING USING WIRELESS BODY AREA NETWORK}

The main reason of death in the world is Cardio-Vascular Disease (CVD), representing 30\% of all global deaths. According to the WHO, worldwide nearly 17.5 million people die due to heart attacks or strokes each year; in 2015, estimated 20 million people will die from CVD . These deaths can often be sttoped with proper health care.

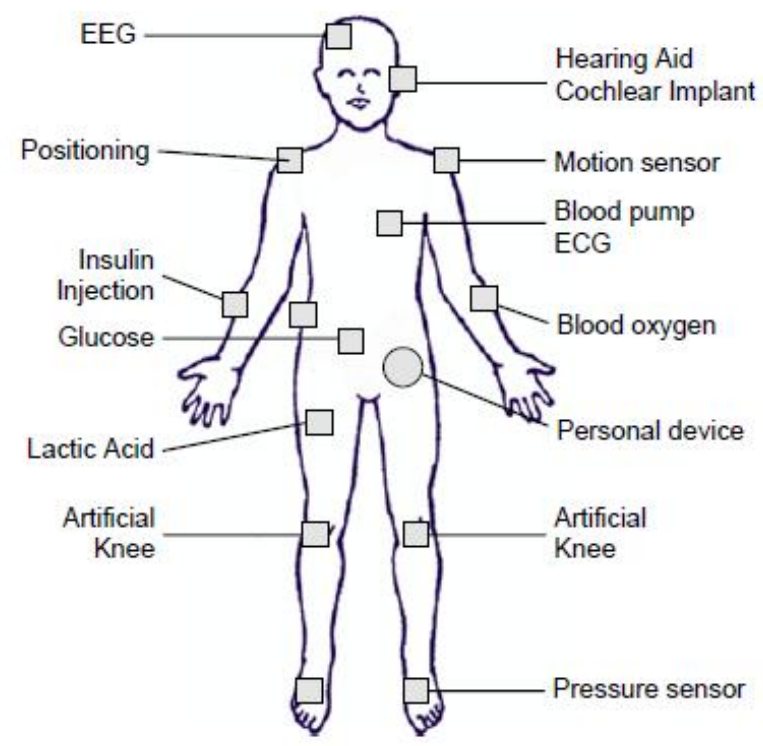

Fig. 2 : Example of patient monitoring in a Wireless Body

\section{Area Network.}

The WBAN directs for example the level of toxics in the air and warns the fire-fighters or soldiers in case of life threatening level is detected. The introduction of a WBAN further facilitate to tune more adequately the training schedules of professional athletes.

\subsection{WBAN Wireless Communication}

Long-life, persistent sensor nodes require efficient power management. With highly integrated electronics, the sensor size and weight becomes dominated by battery selection. An implementation must address conflicting requirements for small size and infrequent battery maintenance, striving for a balance that will maximize user compliance. It is our challenge as designers to minimize sensor power consumption and thus maximize battery life for a given size. In designing our prototype we have held low power consumption as a primary design goal in every component of the system - in processor and technology selection, in managing sensor data, in network organization, and in efficient communications.

Power consumption of the sensor node is dominated by the wireless radio. Nearly $85 \%$ can be attributed to CC2420 controller - even when not actively transmitting. The CC2420, although the lowest power of its kind, still draws $17.4 \mathrm{~mA}$ when transmitting and $19.7 \mathrm{~mA}$ when receiving. In contrast, the MSP430 utilizes $250 \mu \mathrm{A} / \mathrm{MIPS}$ - typically just over 1mA when active. As an example, the MSP430 can execute 100, 000 instructions for the same cost of transmitting a single 40 byte message. With that in mind, power savings can be realized by disabling the radio when not in use as well as reducing the total quantity of transmission - even if extensive computation is required.

\subsubsection{IEEE 802.15.4 and ZigBee}

Our prototype WBAN utilizes the IEEE 802.15.4 compliant CC2420 radio for wireless communications. The IEEE 802.15.4 standard defines communications for nodes in a lowrate wireless personal area network (LR-WPAN) and is well suited for our prototype WBAN. The standard specifies the physical (PHY) layer and data link / media access control (MAC) layer. At the physical layer, IEEE 802.15.4 defines three frequency bands, spread spectrum chip rate, and data encoding [IEEE802.15.4]. ZigBee and IEEE 802.15.4 are cooperating protocol stacks. ZigBee is tightly coupled to 802.15.4 in that the PHY and MAC layers are specified to be IEEE 802.15.4;however, the ZigBee specification details the upper protocol layers - network, application and application sub layer, and security. It specifies network topologies ,routing mechanisms and dynamic discovery and registration of nodes as they enter and exit the network.

\subsubsection{Power Efficient TDMA}

Exploiting the ZigBee star network topology [ZigBee] and an 802.15.4 like super frame, we employed a collision-free Time Division Multiple Access (TDMA) scheme .Fig. 2.3 shows our communication super frame. All communications are in between a sensor node and the network coordinator. Each communication super frame is divided into $50 \mathrm{~ms}$ timeslots used for message transmissions. Each sensor uses its corresponding timeslot to transmit sensor data, command acknowledgements, and event messages. The first timeslot, however, belongs to the network coordinator and is used for transmitting configuration commands from the personal server and sending periodic beacon messages to synchronize other sensors and mark the start of super frames.

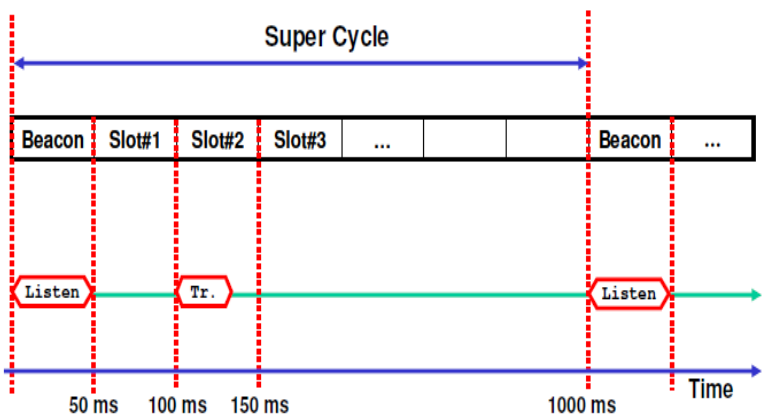

Fig. 3 : Communication super frame and an example of sensor with logical ID=2 
This organization has several advantages. Rather than receiving and transmitting asynchronously, transmissions are scheduled for predetermined time instances, which make communication more deterministic. This approach serves as practical collision avoidance - making more efficient use of the available bandwidth when compared to using only the CC2420 Collision Sense Multiple Access (CSMA) scheme. Most importantly, this allows the sensor node radio to be disabled during inactive time slots. Super frame period and timeslot size are application-specific and must be chosen to balance a tolerable event latency and sensor data bandwidth requirements with low power consumption. Based on a one second super frame and $50 \mathrm{~ms}$ timeslots, the radio on each sensor is active at most $10 \%$ of the time. By disabling the radio during inactive timeslots an average current consumption of just $3.1 \mathrm{~mA}$ can be realized, which achieves 7 times longer battery life compared to a radio that is never disabled. The recorded power profiles for a motion sensor using an environment for real time power monitoring

\section{METHODOLOGY USED}

In this section we characterize our model of a wireless body area network with nodes heterogeneous in their initial amount of energy. We particularly present the setting, the energy model, and how the optimal number of clusters can be computed. Let us assume the case where a percentage of the population of patient nodes is equipped with more energy resources than the rest of the nodes.

Let $m$ be the fraction of the total number of patient nodes $n$, which is equipped with $\alpha$ times more energy than the others. We refer to these powerful nodes as advanced nodes, and the rest $(1-m) \times n$ as normal patient nodes. We assume that all nodes are distributed uniformly over the wireless field. Our main objective is to propose and implement a cluster based energy efficient and stable routing mechanism for wireless body area network in which heads are decided through ratio of energies and random head selection probability is decided through integer linear programming to perform an efficient inter-cluster and intra-cluster communication to transmit data to a base monitoring authority.

The indoor hospital BAN communication devices are categorized in three classes with respect to their available Energy sources. Class 1 devices are directly connected with the power source like Nursing Station Coordinator (NSC). Class 2 devices use the consumable batteries like Medical Display Coordinators (MDCs). BAN Coordinators (BANCs) with the limited energy availability are considered the Class 3 devices. Two channels are used by Class 1 and 2 devices. 802.15.4 is used to communicate with the BANC and 802.11 for Wi Fi. Table below shows the summary of the classes. The NSC database contains the information of all BANCs and MDCs in the BAN peering framework. Initially BANCs search and then connect to the NSC. Each BANC receives the information about its respective peer from the NSC and then starts sending real time BAN data to its respective peer MDC for display.
Table no. 1 parameter settings of summary of the classes in WBAN

\begin{tabular}{|c|c|c|c|c|c|}
\hline $\begin{array}{c}\text { Device } \\
\text { Class }\end{array}$ & $\begin{array}{c}\text { Device } \\
\text { name }\end{array}$ & Power Source & $\begin{array}{c}\text { Number of } \\
\text { Channels }\end{array}$ & $\begin{array}{c}\text { MAC } \\
\text { protocol }\end{array}$ & Mobility \\
\hline 1 & NSC & $\begin{array}{c}\text { Directly } \\
\text { Connected }\end{array}$ & 2 & 802.15 .4 & No \\
\hline 2 & MDC & $\begin{array}{c}\text { Replaceable } \\
\text { batteries }\end{array}$ & 2 & $\begin{array}{c}802.15 .4 \\
802.11\end{array}$ & Yes \\
\hline 3 & BANC & $\begin{array}{c}\text { imimited energy } \\
\text { available }\end{array}$ & 1 & 802.15 .4 & Yes \\
\hline
\end{tabular}

\subsection{Hierarchically Clustered Model for Communication}

We assumes a wireless body area network that is hierarchically clustered. Our proposed algorithm maintains such clustering hierarchy. In our protocol, the clusters are reestablished in each "round." New cluster heads are elected in each round and as a result the load is well distributed and balanced among the nodes of the network.

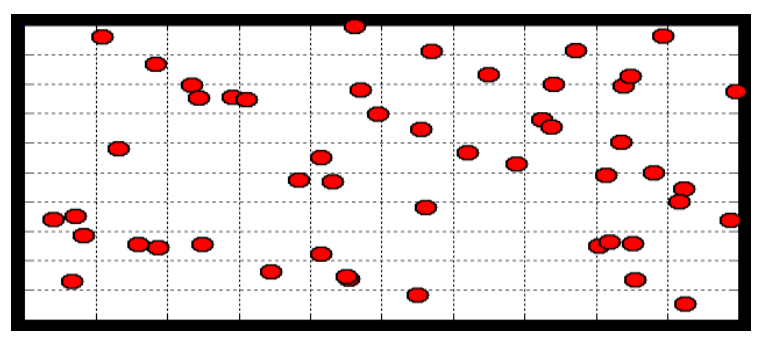

Fig 4 : Patient Node placement in network environment (for 50 sensors placed randomly in the field of $\mathbf{1 0 0 0 0}$ meter square area)

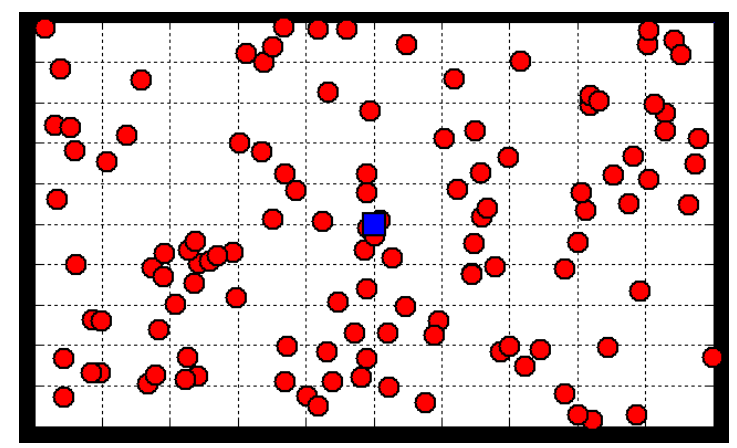

Fig 5 : Patient Node placement in network environment and base station placed at the Centre of field (for 100 patient sensors placed randomly in the field of 10000 meter square area)

\subsection{Prime Clustering Based on Attributes}

Previous work have studied either by simulation or analytically the optimal probability of a node being elected as a cluster head as a function of spatial density when nodes are uniformly distributed over the sensor field. According to the radio energy dissipation model illustrated in Figure, 4.4 in order to achieve an acceptable Signal-to-Noise Ratio (SNR) in transmitting an L-bit message over a distance $d$, the energy expended by the radio is given by: 


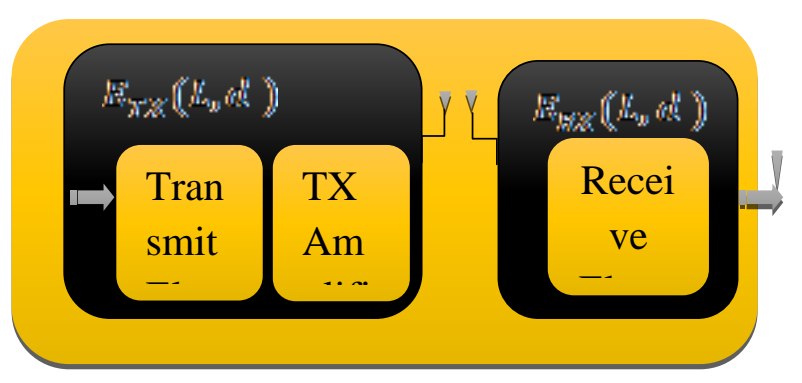

Fig 6 : Radio Energy Dissipation Model

$$
E_{T 2}(l, d)=\left\{\begin{array}{c}
L \cdot E_{e l e c}+L \cdot \in_{f s} \cdot d^{2} \text { if } d \leq d_{0} \\
L . E_{\text {elec }}+L \cdot \in_{m p} \cdot d^{4} \text { if } d>d_{0}
\end{array}\right\}
$$

Here $E_{\text {elec }}$ is the energy dissipated per bit to run the transmitter or the receiver circuit, $\in_{f s}$ and $\epsilon_{m p}$ depend on the transmitter amplifier model we use, and $d$ is the distance between the sender and receiver. By equating the two expressions at $d=d 0$, we have $d_{0}=\sqrt{\epsilon_{f s} / \epsilon_{m p}}$. То receive an L-bit message the radio expends $E_{R x}=$ L. $E_{\text {elec }}$. This radio model Help will Help us to calculate the amount of dissipated energy after every round based on distance vector based calculation.

\subsection{Procedural Steps}

First section is network initialization, in this phase we have to decide the network parameters, like filed area, number of devices, device parameters After the initialization and setup phase completed, the transmission phase is starts, in this phase, initially we calculate and update the energy values of every device and it will update at every transmission round.

Table no. 2 Parameter setting of the first order radio model

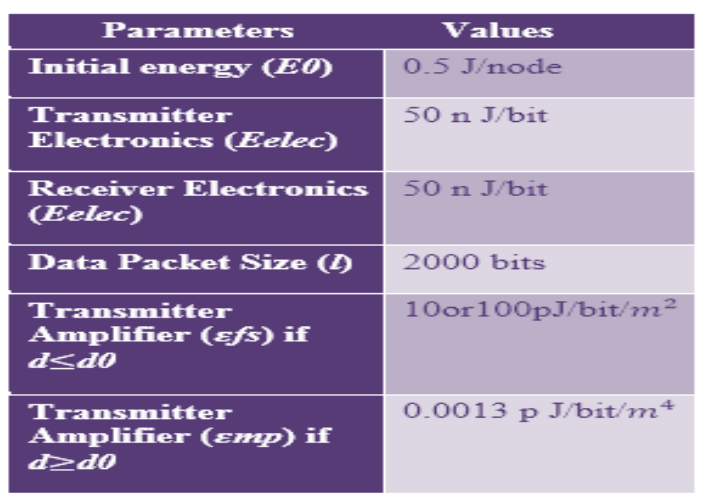

The Clustering and routing procedure endure till the network devices alive, the devices with a proper energy levels are selected as cluster head one after another every round. After each transmission round, device's residual energy is calculated with the radio energy model for wireless communication network, this helps us in deciding a cluster head node to continue transmission in the next transmission round. In case of research work in wireless network, system efficiency can be calculated from the relation of input and output data packets. Hence the throughput, end to end delay, packet delivery fraction ratio, and network lifetime are the best suited parameters to show research efficiency.

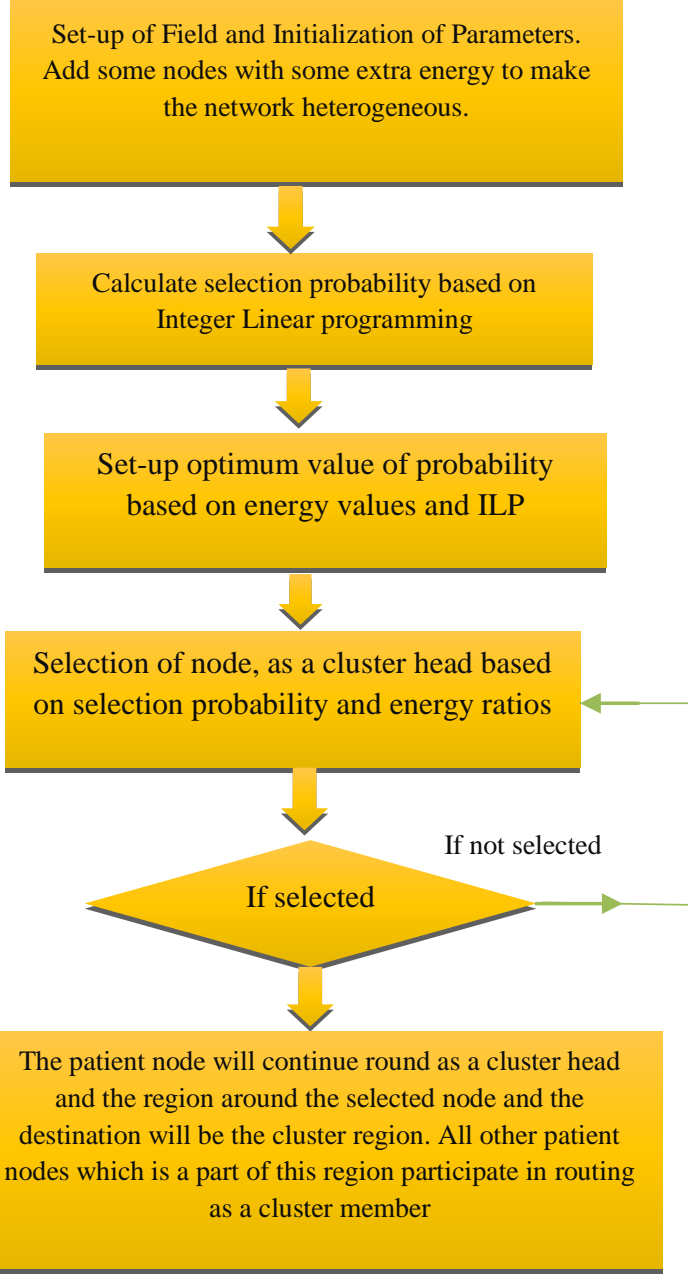

Fig 7 : Communication steps and Flow chart for the procedural phases progressive

\subsection{Algorithm in Flow}

\section{Network Initialization}

// A random Field created and nodes are randomly placed, every node contains a specified amount of energy

\section{Setup Phase}

// Bisection between nodes through random modelling, and path-cost calculated through distance vector estimation

\section{Transmission phase}

For 1: 1: Maximum transmission rounds

Update Average Energy with respect to rounds,

$$
E_{\text {avg }}=E_{\text {tot }} \times\left(\frac{1-\frac{r}{R \max }}{n}\right)
$$

Check for Dead Criteria at every Start Up

For $\boldsymbol{i}=1: n$

Check Nodes \& Update Flags

Update Dead \& Alive Statistics

End

For $i=1: n$ 
If $E_{a v g}>0$ (means checking if there's maximum round reaches)

Calculate probability of selection of Cluster Head

$P(i)=P_{I L P} \times n \times \frac{E_{\text {int }}}{E_{\text {tot }}} \times \frac{E_{\text {curr }}}{E_{\text {avg }}}$

Here, $P_{I L P}$ is calculate through the integer linear programming, based upon the optimum solution of our algorithm, $E_{\text {int }}$ initially supplied energy with respect to total energy $E_{\text {tot }}$ and $E_{\text {curr }}$ is current resource status with respect to average energy in network $E_{\text {avg }}$

NOTE: The best patient node select within the network whose $\frac{E_{\text {int }}}{E_{\text {tot }}}$ and $\frac{E_{\text {curr }}}{E_{\text {avg }}}$ ratio is high, also its selection probability is also high which is decided through $P_{I L P}$

If $(\boldsymbol{S}(\boldsymbol{i}) . \boldsymbol{E}>0)$

Temp = a temporary random number allotted to every node

If temp $<=T\left(s_{i}\right)$

Where, $T\left(s_{i}\right)=\left\{\begin{array}{l}\frac{P_{i}}{1-P_{i}\left(\operatorname{rmod} \frac{1}{P_{i}}\right)} \quad \text { if } \in G \\ 0 \quad \text { otherwise }\end{array}\right.$

Update Packet Counter as per selection as the set up phase completed

Update Clusters Counter as per cluster head Selection in patient network

(We denote the methodology as cluster based because we first select a cluster head based on energy level and then assuming the region around it as a cluster and the selected sub-destination will be the head of that cluster)

Update the selected node number value as an id number for cluster region formed

\section{Update Cluster Area}

(The Cluster Area is the area between the selected cluster head and the base station based on distance Vector calculated between them, and the devices between these Regions are called as cluster member)

Data Transmission from Selected Cluster head based on Distance Vector Calculated

$$
S(i) . E=\left\{\begin{array}{l}
l E_{d e c}+l \varepsilon_{f x} d^{2}, d<d_{0} \\
l E_{d e c}+l \varepsilon_{f x} d^{4}, d \geq d_{0}
\end{array}\right.
$$

Update Residual Energy for the selected cluster head from the formula above

(All the Nodes \& Future Cluster heads are not active during this time or we can say that they are in sleep mode)

End

End

End

\section{RESULTS}

In this section, the proposed cluster-based WBAN technique is evaluated with respect to energy consumption of nodes. All experiments were performed using MATLAB (Math works, Natick, MA, USA).
First of all we will develop a GUI using MATLAB for generating the desired results,

Suppose we are performing our analysis with 100 patients, so set the no. of patients 100 , which has to be sense. Next we will create a field for wireless network, a 10000 sq meter wireless field will be created. After creating field placing of patients and base station will be done, shown below.

After placing of patients and base station next we have to check mobility of the patients. In next step we will calculate the distance between the patients or we can say that we will do distance vector calculation. These four process comes under network initialization.

Next we had done network simulation. In network simulation we had optimized probability with MILP and then executed proposed protocol

Next we had done coparative analysis between routing in base paper and LEACH based routing,and after doing that we can show a comparative study for energy efficient system. The results shown as follows:

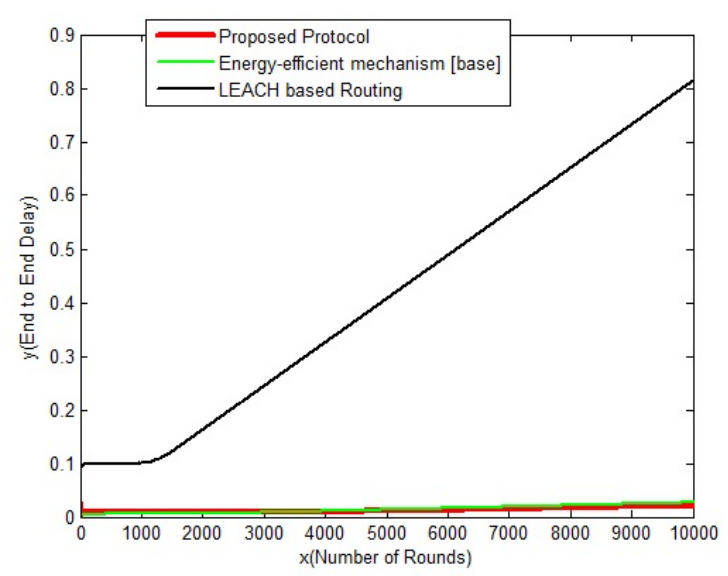

Fig 8 : Network Delay

This comparative figure shows the network delay between proposed protocol, energy- efficient mechanism and LEACH based routing.

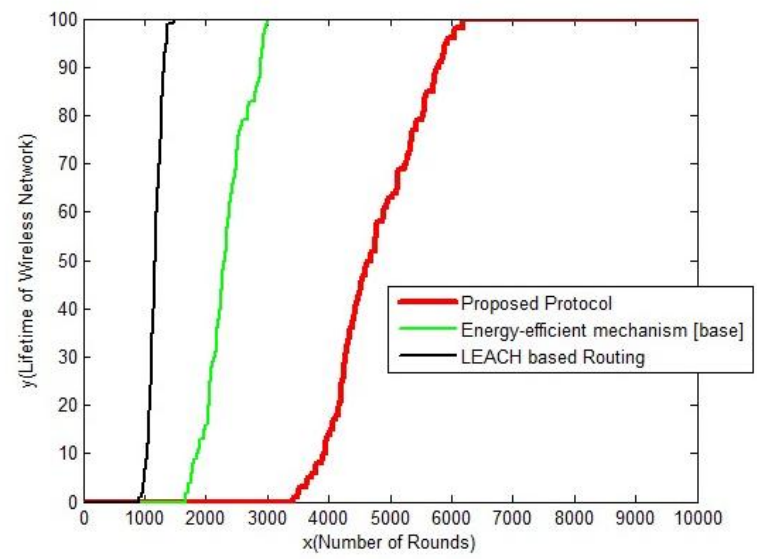

Fig : 9 Network Lifetime

This comparative figure shows the network lifetime between proposed protocol, energy- efficient mechanism and LEACH based routing. 


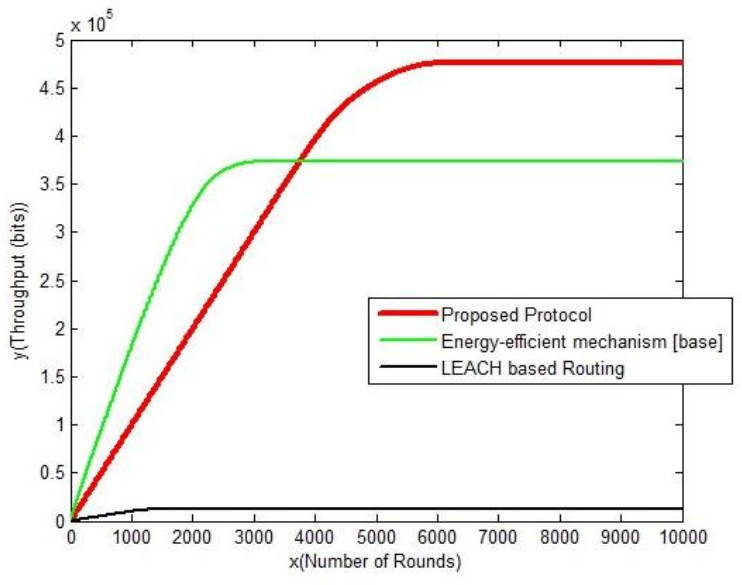

Fig 10 : Network Throughtput (bits)

This comparative figure shows the network Throughtput between proposed protocol, energy- efficient mechanism and LEACH based routing.

This graphical presentation is further evaluated in tabular form. Each section is presented separately i.e with respect to proposed protocol, energy efficient mechanism (which is considered as base of my research) and LEACH baesd protocol.

In our project we had taken rounds till 10,000 but as we can see after 6000 rounds there is no such changes has been evaluated. The table contains life time of wireless network and throughput (bits). All the three tables is based on the same Contains.

Table no. 3 is the analysis based proposed protocol which contains life time of network and throughputs (bits)

Table No. 3

Result analysis based on proposed protocol LT - Lifetime Network, W/N- Wireless Network, PPProposed protocol

\begin{tabular}{|c|c|c|c|}
\hline \multicolumn{3}{|c|}{ 100 Patients } \\
\cline { 3 - 4 } Sr. No. & \multirow{2}{*}{ Rounds } & \multicolumn{2}{c|}{ PP } \\
\cline { 3 - 4 } & & LT of W/N & Throughput(bits) \\
\hline 1 & 500 & 00 & $4.99 \mathrm{e}+004$ \\
\hline 2 & 1000 & 00 & $9.99 \mathrm{e}+004$ \\
\hline 3 & 1500 & 00 & $1.499 \mathrm{e}+005$ \\
\hline 4 & 2000 & 00 & $1.999 \mathrm{e}+005$ \\
\hline 5 & 2500 & 00 & $2.499 \mathrm{e}+005$ \\
\hline 6 & 3000 & 07 & $2.999 \mathrm{e}+005$ \\
\hline
\end{tabular}

\begin{tabular}{|c|c|c|c|}
\hline 7 & 3500 & 07 & $3.49 \mathrm{e}+005$ \\
\hline 8 & 4000 & 21 & $3.927 \mathrm{e}+005$ \\
\hline 9 & 4500 & 43 & $4.264 \mathrm{e}+005$ \\
\hline 10 & 5000 & 67 & $4.487 \mathrm{e}+005$ \\
\hline 11 & 5500 & 86 & $4.6 \mathrm{e}+005$ \\
\hline 12 & 6000 & 98 & $4.63 \mathrm{e}+005$ \\
\hline
\end{tabular}

Table No. 4

Result analysis for energy efficient mechanism LT - Lifetime Network, W/N- Wireless Network, EEM Energy efficient mechanism

\begin{tabular}{|c|c|c|c|}
\hline \multirow{2}{*}{ Sr. No. } & \multirow{2}{*}{ Rounds } & \multicolumn{2}{|c|}{ EEM (Base) } \\
\cline { 3 - 4 } & & LT of W/N & Throughput(bits) \\
\hline 1 & 500 & 00 & $9.354 \mathrm{e}+004$ \\
\hline 2 & 1000 & 00 & $1.81 \mathrm{e}+005$ \\
\hline 3 & 1500 & 00 & $2.615 \mathrm{e}+005$ \\
\hline 4 & 2000 & 18 & $3.275 \mathrm{e}+005$ \\
\hline 5 & 2500 & 72 & $3.612 \mathrm{e}+005$ \\
\hline 6 & 3000 & 98 & $3.697 \mathrm{e}+005$ \\
\hline 7 & 3500 & 100 & $3.697 \mathrm{e}+005$ \\
\hline 8 & 4000 & 100 & $3.697 \mathrm{e}+005$ \\
\hline 9 & 4500 & 100 & $3.697 \mathrm{e}+005$ \\
\hline 10 & 5000 & 100 & $3.697 \mathrm{e}+005$ \\
\hline 11 & 5500 & 100 & $3.697 \mathrm{e}+005$ \\
\hline 12 & 6000 & 100 & $3.697 \mathrm{e}+005$ \\
\hline
\end{tabular}

Table No. 5

Result analysis for LEACH based protocol LT - Lifetime Network, W/N- Wireless Network

\begin{tabular}{|c|c|c|c|}
\hline \multicolumn{3}{|c|}{100 Patients } \\
\cline { 3 - 4 } Sr. No. & \multirow{2}{*}{ Rounds } & \multicolumn{2}{|c|}{ LEACH based protocol } \\
\cline { 3 - 4 } & & LT of W/N & Throughput(bits) \\
\hline 1 & 500 & 00 & 5015 \\
\hline 2 & 1000 & 01 & $1.001 \mathrm{e}+004$ \\
\hline 3 & 1500 & 100 & $1.218 \mathrm{e}+004$ \\
\hline 4 & 2000 & 100 & $1.218 \mathrm{e}+004$ \\
\hline 5 & 2500 & 100 & $1.218 \mathrm{e}+004$ \\
\hline 6 & 3000 & 100 & $1.218 \mathrm{e}+004$ \\
\hline 7 & 3500 & 100 & $1.218 \mathrm{e}+004$ \\
\hline 8 & 4000 & 100 & $1.218 \mathrm{e}+004$ \\
\hline 9 & 4500 & 100 & $1.218 \mathrm{e}+004$ \\
\hline 10 & 5000 & 100 & $1.218 \mathrm{e}+004$ \\
\hline 11 & 5500 & 100 & $1.218 \mathrm{e}+004$ \\
\hline 12 & 6000 & 100 & $1.218 \mathrm{e}+004$ \\
\hline
\end{tabular}

Table no. 6

Combined result analysis on 100 patients

LT - Lifetime Network, W/N- Wireless Network, PP- Proposed protocol, EEM - Energy efficient mechanism

\begin{tabular}{|c|c|c|c|c|c|c|c|}
\hline \multicolumn{2}{|c|}{$\begin{array}{c}\text { 100 Patients } \\
\text { Sr. } \\
\text { No. }\end{array}$} & \multirow{2}{*}{ Rounds } & \multicolumn{2}{|c|}{ PP } & \multicolumn{2}{c|}{ EEM (base) } & \multicolumn{2}{c|}{ LEACH based protocol } \\
\cline { 3 - 9 } & LT of W/N & $\begin{array}{c}\text { Throughput } \\
\text { (bits) }\end{array}$ & LT of W/N & Throughput (bits) & \multicolumn{2}{|c|}{ LT of W/N } & Throughput (bits) \\
\hline 1 & 500 & 00 & $4.99 \mathrm{e}+004$ & 00 & $9.354 \mathrm{e}+004$ & 00 & 5015 \\
\hline 2 & 1000 & 00 & $9.99 \mathrm{e}+004$ & 00 & $1.81 \mathrm{e}+005$ & 01 & $1.001 \mathrm{e}+004$ \\
\hline 3 & 1500 & 00 & $1.499 \mathrm{e}+005$ & 00 & $2.615 \mathrm{e}+005$ & 100 & $1.218 \mathrm{e}+004$ \\
\hline 4 & 2000 & 00 & $1.999 \mathrm{e}+005$ & 18 & $3.275 \mathrm{e}+005$ & 100 & $1.218 \mathrm{e}+004$ \\
\hline 5 & 2500 & 00 & $2.499 \mathrm{e}+005$ & 72 & $3.612 \mathrm{e}+005$ & 100 & $1.218 \mathrm{e}+004$ \\
\hline 6 & 3000 & 07 & $2.999 \mathrm{e}+005$ & 98 & $3.697 \mathrm{e}+005$ & 100 & $1.218 \mathrm{e}+004$ \\
\hline
\end{tabular}




\begin{tabular}{|c|c|c|c|c|c|c|c|}
\hline 7 & 3500 & 07 & $3.49 \mathrm{e}+005$ & 100 & $3.697 \mathrm{e}+005$ & 100 & $1.218 \mathrm{e}+004$ \\
\hline 8 & 4000 & 21 & $3.927 \mathrm{e}+005$ & 100 & $3.697 \mathrm{e}+005$ & 100 & $1.218 \mathrm{e}+004$ \\
\hline 9 & 4500 & 43 & $4.264 \mathrm{e}+005$ & 100 & $3.697 \mathrm{e}+005$ & 100 & $1.218 \mathrm{e}+004$ \\
\hline 10 & 5000 & 67 & $4.487 \mathrm{e}+005$ & 100 & $3.697 \mathrm{e}+005$ & 100 & $1.218 \mathrm{e}+004$ \\
\hline 11 & 5500 & 86 & $4.6 \mathrm{e}+005$ & 100 & $3.697 \mathrm{e}+005$ & 100 & $1.218 \mathrm{e}+004$ \\
\hline 12 & 6000 & 98 & $4.63 \mathrm{e}+005$ & 100 & $3.697 \mathrm{e}+005$ & 100 & $1.218 \mathrm{e}+004$ \\
\hline
\end{tabular}

Table No. 7

Combined result analysis on 150 patients

LT - Lifetime Network, W/N- Wireless Network, PP- Proposed protocol, EEM - Energy efficient mechanism

\begin{tabular}{|c|c|c|c|c|c|c|c|}
\hline \multicolumn{8}{|c|}{150 Patients } \\
\hline \multirow{2}{*}{$\begin{array}{l}\text { Sr. } \\
\text { No. }\end{array}$} & \multirow[b]{2}{*}{ Rounds } & \multicolumn{2}{|c|}{ PP } & \multicolumn{2}{|c|}{ EEM (base) } & \multicolumn{2}{|c|}{ LEACH based protocol } \\
\hline & & LT of W/N & $\begin{array}{c}\text { Throughput } \\
\text { (bits) }\end{array}$ & LT of W/N & Throughput (bits) & LT of W/N & Throughput (bits) \\
\hline 1 & 500 & 00 & $7.455 \mathrm{e}+004$ & 00 & $1.405 \mathrm{e}+005$ & 00 & 7509 \\
\hline 2 & 1000 & 00 & $1.496 \mathrm{e}+005$ & 00 & $2.72 \mathrm{e}+005$ & 03 & $1.502 \mathrm{e}+004$ \\
\hline 3 & 1500 & 00 & $2.246 \mathrm{e}+005$ & 00 & $3.929 \mathrm{e}+005$ & 150 & $1.825 \mathrm{e}+004$ \\
\hline 4 & 2000 & 00 & $2.996 \mathrm{e}+005$ & 26 & $4.926 \mathrm{e}+005$ & 150 & $1.825 \mathrm{e}+004$ \\
\hline 5 & 2500 & 00 & $3.746 e+005$ & 98 & $5.46 \mathrm{e}+005$ & 150 & $1.825 \mathrm{e}+004$ \\
\hline 6 & 3000 & 00 & $4.496 e+005$ & 147 & $5.585 \mathrm{e}+005$ & 150 & $1.825 \mathrm{e}+004$ \\
\hline 7 & 3500 & 03 & $5.24 \mathrm{e}+005$ & 150 & $5.585 \mathrm{e}+005$ & 150 & $1.825 \mathrm{e}+004$ \\
\hline 8 & 4000 & 20 & $5.948 \mathrm{e}+005$ & 150 & $5.585 \mathrm{e}+005$ & 150 & $1.825 \mathrm{e}+004$ \\
\hline 9 & 4500 & 61 & $6.526 \mathrm{e}+005$ & 150 & $5.585 \mathrm{e}+005$ & 150 & $1.825 \mathrm{e}+004$ \\
\hline 10 & 5000 & 91 & $6.898 \mathrm{e}+005$ & 150 & $5.585 \mathrm{e}+005$ & 150 & $1.825 \mathrm{e}+004$ \\
\hline 11 & 5500 & 113 & $7.13 e+005$ & 150 & $5.585 \mathrm{e}+005$ & 150 & $1.825 \mathrm{e}+004$ \\
\hline 12 & 6000 & 138 & $7.257 e+005$ & 150 & $5.585 \mathrm{e}+005$ & 150 & $1.825 \mathrm{e}+004$ \\
\hline
\end{tabular}

\section{CONCLUSION}

WBANs play an vital role in providing better healthcare services by using uninterrupted and real-time monitoring of health information. Before using WBANs on real test beds, one must address the consumption of energy requirements of WBANs as these requirements increase the usability and usefulness of WBANs. The technique conferred in this paper uses clustering. Introducing secure cluster topology formation to WBAN communication makes the communication energyefficient and increases the life of network. In this paper we compared the proposed protocol with the LEACH based scheme and energy efficient mechanism. The results of our analysis show that the proposed scheme produces much better results in terms of energy consumption, cluster formation, and network lifetime.

The analysis of our proposed WBAN communication scheme in terms of energy efficiency, the cluster formation process in WBAN communication brings energy efficiency to the project. The proposed scheme is highly suitable for WBAN applications in terms of energy.

\section{ACKNOWLDGEMENT}

The authors would like to extend their sincere thanks to the Electronics \& Communication department, NIIST Bhopal (m.p).

\section{REFERENCES}

[1] Shariar Imtiaz, November 2013 "Improved Adaptive Routing for Multihop IEEE 802.15.6 Wireless Body Area Networks", I.J. Intelligent Systems and Applications, 2013, 12, 64-71Published Online in MECS (http://www.mecs-press.org/).

[2] Xiuming Zhu, 2011 "MBStar: A Real-time Communication Protocol for Wireless Body AreaNetworks", 23rd Euromicro Conference onReal time Systems (ECRTS).

[3] Ahmed Shoeb Al Hasan, December 2013 "Reliable ECG Signals Based on GMM for BodyArea Network", Volume 2 Issue 12

[4] Meenakshi Bansal, March 2011 "Use of Relay Nodes in Body Area Sensor Network for Reducing Power Consumption", IJCST Vol. 2, Issue 1.

[5] Baozhi Chen, Proceedings of the 2010 "Patient Vital Signs Monitoring using Wireless Body Area Networks", IEEE 36th Annual Northeast Bioengineering Conference.

[6] Veerpal Kaur, March 2011 "Performance Evaluation of Binary Modulations throughWireless Body Area Network Channel", Volume 18- No.3.

[7] Prakashgoud Patil, October 2013 "Fuzzy Logic based Health Care System using Wireless Body Area Network", Volume 80 - No 12. 
[8] Laura Galluccio, 2012 "Challenges and Implications of Using Ultrasonic Communications in Intra-body Area Networks", 9th Annual Conference on Wireless OnDemand Network Systems and Services (WONS)

[9] Mohammed Mana, November 2009, "SEKEBAN (Secure and Efficient Key Exchange for wireless BodyArea Network)", Vol. 12.

[10] Jinhyuk Kim,June, 2012 “A Dynamic Duty Cycle MAC Algorithm for Wireless Body Area Networks", Vol. 4, No. 2.

[11] Ali Peiravi,2010 "Connectance and Reliability Computation of Wireless Body Area Networks using Signal Flow Graphs”, Life Science Journal.

[12] Kyeong Hur, March, 2013 “A WBAN Beacon Structure for Wireless USB Protocol Adaptation”, Vol. 8, No. 2.

[13] Ali Peiravi, 2010 "Reliability of Wireless Body Area Networks used for Ambulatory Monitoring and Health Care", Life Science Journal.
[14] Emil Jovanov,2005 “A wireless body area network of intelligent motion sensors for computer assisted physical rehabilitation", Journal of Neuro Engineering and Rehabilitation.

[15] Chris Otto, 2006 "System Architecture of a Wireless Body Area Sensor Network for Ubiquitous Health Monitoring", Journal of Mobile Multimedia, Vol. 1, No.4.

[16] Chris A. Otto,2006 "A WBAN-based System for Health Monitoring at Home", 3rd IEEE/EMBS International Summer School on Medical Devices and Biosensors.

[17] Gabriel E. Arrobo, 2011 "New Approaches to Reliable Wireless Body Area Networks", COMCAS.

[18] Jocelyne Elias, "Energy-aware Topology Design for Wireless BodyArea Networks", IEEE ICC 2012 Selected Areas in Communications Symposium. 